\title{
A atuação da terapia ocupacional com mulheres com câncer de mama em cuidados paliativos*
}

\section{Role of occupational therapy to women with breast cancer in palliative care}

\author{
Natália Cintra Faria ${ }^{1}$, Marysia Mara Rodrigues do Prado De Carlo²
}

http://dx.doi.org/10.11606/issn.2238-6149.v26i3p418-427

Faria NC, De Carlo MRP. A atuação da terapia ocupacional com mulheres com câncer de mama em cuidados paliativos. Rev Ter Ocup Univ São Paulo. 2015 set.-dez.;26(3):418-27.

RESUMO: Estudo retrospectivo, descritivo, com abordagem qualitativa, baseado em análise documental, para revisão dos dados sociodemográficos e clínicos e dados referentes aos atendimentos de Terapia Ocupacional. O objetivo é identificar e categorizar as intervenções do terapeuta ocupacional com mulheres com câncer de mama atendidas pelo serviço de Cuidados Paliativos de um hospital público, universitário, de alta complexidade, localizado no interior do Estado de São Paulo. O projeto foi aprovado pelo Comitê de Ética em Pesquisa (Processo no 12559/2013). A casuística inicial foi composta por 49 mulheres com diagnóstico de câncer ginecológico, dentre as quais foram identificadas 27 com câncer de mama; 23 delas haviam sido atendidas pela terapeuta ocupacional e compuseram a amostra final deste estudo. Os dados e os procedimentos realizados foram analisados por estatística descritiva. intervenções abrangeram tanto o paciente como sua família; envolveram diferentes procedimentos, como acolhimento e suporte familiar, orientações sobre estratégias de enfrentamento, conservação de energia, estímulo à independência e autonomia para as AVDs e AIVDs e atividades significativas. A Terapia Ocupacional possibilita a construção de espaços saudáveis de vida e de potência, criação e singularidade nos Cuidados Paliativos.

DESCRITORES: Neoplasias da mama; Terapia ocupacional; Cuidados paliativos.
Faria NC, De Carlo MRP. Role of occupational therapy to women with breast cancer in palliative care. Rev Ter Ocup Univ São Paulo. 2015 Sept.-Dec.;26(3):418-27.

\begin{abstract}
A retrospective, descriptive study with a qualitative approach, based on document analysis, to review the socio-demographic data, clinical and referring to Occupational Therapy visits. The goal is to identify and to categorize the interventions of occupational therapist with women with breast cancer, served by the Palliative Care Service of a public hospital, university, high complexity, located in the state of São Paulo. The project was approved by the ethics committee in research (Process n. 1259 12013). The initial sample was composed of 49 women diagnosed with gynecological cancer, of which 27 have been identified breast cancer; 23 of them had been met by the occupational therapist and composed the final sample. Data and procedures were analyzed using descriptive statistics. Interventions covered both the patient and his family; involved different procedures, such as care and family support, guidance on coping strategies, energy conservation, encouraging independence and autonomy for the ADL and IADL and meaningful activities. Occupational therapy enables the construction of healthy environments and power, creation and uniqueness in Palliative Care.
\end{abstract}

KEYWORDS: Breast cancer; Occupational therapy; Palliative care.

\footnotetext{
* Trata-se de um estudo que foi apresentado para conclusão do Programa de Aprimoramento Profissional de "Terapia Ocupacional Hospitalar". Fonte de Financiamento: Programa de Aprimoramento Profissional de "Terapia Ocupacional Hospitalar". Secretaria de Estado da Saúde, Fundação do Desenvolvimento Administrativo - SES/FUNDAP.

Procedimentos éticos: foram cumpridos (Resolução CNS 466/12.) e a execução da pesquisa autorizada pelo Comitê de Ética em Pesquisa do Hospital das Clínicas da Faculdade de Medicina de Ribeirão Preto (Processo HCRP 12559 / 2013).

1. Terapeuta Ocupacional graduada pela FMRP-USP,Terapeuta Ocupacional do Hospital das Clínicas da Faculdade de Medicina de Ribeirão Preto e mestranda pelo Programa de Pós-graduação de Enfermagem em Saúde Pública da Escola de Enfermagem de Ribeirão Preto, Universidade de São Paulo. E-amil: nataliacintrafaria@yahoo.com

2. Professora Doutora do Curso de Terapia Ocupacional, Departamento de Neurociências e Ciências do Comportamento da Faculdade de Medicina de Ribeirão Preto e do Programa de Pós-graduação de Enfermagem em Saúde Pública da Escola de Enfermagem de Ribeirão Preto, Universidade de São Paulo. E-mail: marysia@fmrp.usp.br

Endereço para correspondência: Natália Cintra Faria. Rua Pirajá da Silva, 201. Alto da Boa Vista. Ribeirão Preto, SP, Brasil. CEP: 14025-570. E-mail: nataliacintrafaria@yahoo.com
} 


\section{INTRODUÇÃO}

$\mathrm{O}$ câncer é um crescente problema de saúde pública mundial. O Instituto Nacional do Câncer (INCA) aponta que em 2030, a carga mundial estimada será de 21,4 milhões de casos novos de câncer e 13,2 milhões de mortes por câncer, em consequência do crescimento e do envelhecimento da população, bem como da redução na mortalidade infantil e nas mortes por doenças infecciosas em países em desenvolvimento ${ }^{1}$

No Brasil, as estimativas para o ano de 2014, válidas também para o ano de 2015, apontam a ocorrência de aproximadamente 576.000 casos novos de câncer, incluindo os casos de pele não melanoma. $\mathrm{O}$ câncer de pele do tipo não melanoma (182 mil casos novos em 2015) é o mais incidente na população brasileira, seguido pelos tumores de próstata (69 mil), mama feminina (57 mil), cólon e reto (33 mil), pulmão (27 mil), estômago (20 mil) e colo do útero (15 mil). Sem considerar os casos de câncer de pele não melanoma, estimam-se 395 mil casos novos de câncer, 204 mil para o sexo masculino e 190 mil para sexo feminino. Em homens, os tipos mais incidentes serão os cânceres de próstata, pulmão, cólon e reto, estômago e cavidade oral; e, nas mulheres, os de mama, cólon e reto, colo do útero, pulmão e glândula tireoide'.

O câncer de mama ocorre tanto em homens quanto em mulheres, sendo, porém, o tipo de câncer ginecológico mais comum no sexo feminino e raro no sexo masculino. A maior incidência se dá em mulheres com idade entre 45 e 55 anos, embora o número de casos em mulheres com idade entre 30 e 40 anos venha aumentando. O tumor pode se propagar para os linfonodos regionais, principalmente para a cadeia axilar, que podem ser facilmente palpáveis em casos avançados de câncer de mama. Outros locais de disseminação linfática podem incluir linfonodos interpeitorais, supraclaviculares, mamários internos e axilares do lado oposto (metástase à distância) ${ }^{2}$.

Há diversos fatores de risco para o câncer de mama, tais como uso de estrogênio exógeno, história familiar positiva, menarca precoce, menopausa tardia, primeira gestação a termo após os 25 anos, nuliparidade, dieta rica em gordura, uso de contraceptivos orais e idade avançada são exemplos deles. A amamentação, prática de atividade física e alimentação saudável, com a manutenção do peso corporal estão associadas a um menor risco de desenvolver esse tipo de câncer ${ }^{2,3}$.

O câncer de mama é uma das principais causas de morte entre as mulheres no mundo ocidental e é o câncer que mais causa mortes entre as mulheres no Brasil - cerca de 11 mil óbitos por ano ${ }^{3}$. Apesar de ser considerado um tipo de câncer de bom prognóstico, se diagnosticado e tratado oportunamente, as taxas de mortalidade por câncer da mama continuam elevadas no Brasil, muito provavelmente porque a doença ainda tem sido diagnosticada em estadios avançados $^{2}$. A sobrevida média após cinco anos na população de países desenvolvidos tem apresentado um discreto aumento, cerca de $85 \%$ das mulheres tem melhor sobrevida. Entretanto, nos países em desenvolvimento, a sobrevida permanece com valores entre 50\% e $60 \%{ }^{3}$.

Dada a complexidade biológica do câncer de mama, a literatura coloca que não há conduta única para o tratamento. Dependendo do caso, podem ser indicadas: radioterapia, quimioterapia, hormonioterapia e mastectomia, de forma associada ou isolada ${ }^{4}$. Muitas são as dificuldades enfrentadas em decorrência do câncer e dos efeitos colaterais dos tratamentos, como dor e dificuldades físicas ${ }^{5}$.

A mastectomia pode alterar a autoimagem da mulher e provocar limitações no seu desempenho funcional e ocupacional. Uma vez que o câncer de mama apresenta curso prolongado podem ocorrer prejuízos em sua independência, o individuo necessita de um programa de tratamento que envolva a realização de exercícios com o membro superior homolateral à mama mastectomizada e outros cuidados como evitar exposição ao calor, evitar carregar peso excessivo com o mesmo. Se esses não forem realizados corretamente, poderão ocorrer outros comprometimentos, como o linfedema ${ }^{4,5}$.

Além disso, a mulher mastectomizada pode vivenciar vários tipos de luto, vergonha em lidar com o próprio corpo e dificuldades ao retomar a sua vida ocupacional $^{5}$. Preconceito, sentimentos de impotência e frustração, ansiedade, depressão e o próprio temor da doença podem comprometer o cotidiano da mulher com câncer de mama, além de prejudicar suas relações sociofamiliares $^{6-9}$.

Assim, há necessidade de estabelecer medidas efetivas para aumentar a qualidade de vida e do tratamento do câncer, principalmente quando não há possibilidades de um tratamento modificador da doença (quando a pessoa está fora de possibilidades de cura). Dessa maneira, ganha importância a filosofia dos Cuidados Paliativos, que deve ser entendida como uma política pública, dentro de um modelo de atenção integral a saúde.

\section{Cuidados paliativos oncológicos e terapia ocupacional}

As ações em Cuidados Paliativos tem se tornado uma importante ferramenta para o cuidado à saúde dos indivíduos acometidos por doenças crônicas degenerativas 
potencialmente fatais e as consequências do adoecimento ${ }^{10}$. De acordo com a Organização Mundial da Saúde (OMS), os Cuidados Paliativos podem ser conceituados como:

"conjunto de medidas capazes de prover uma melhor qualidade de vida ao doente portador de uma doença que ameace a continuidade da vida e seus familiares através do alívio da dor e dos sintomas estressantes, utilizando uma abordagem que inclui o suporte emocional, social e espiritual aos doentes e seus familiares desde o diagnóstico da doença ao final da vida e estendendo-se ao período de luto (p.115)" ${ }^{11}$.

O término de uma terapia curativa para o câncer não significa o final de um tratamento ativo, mas, sim, mudanças em focos de tratamento. Os cuidados paliativos são eminentemente ativos e devem ser aplicados precocemente, no curso da doença, em conjunto com o tratamento oncológico modificador da doença, tornando-se gradualmente um tratamento exclusivo de pacientes em fase avançada até sua morte e também de sua família, inclusive no período de luto ${ }^{11}$.

A prática dos Cuidados Paliativos no Brasil está se ampliando e os trabalhos apresentados sobre o papel da Terapia Ocupacional nos Cuidados Paliativos têm mostrado que esta tem papel significativo na identificação de necessidades e apoio aos pacientes, particularmente aqueles com doenças em fase avançada ${ }^{12}$.

O papel do terapeuta ocupacional em cuidados paliativos está associado à habilidade de observar, escutar e trabalhar com cada paciente em sua individualidade, para alcançar seus objetivos particulares, assegurando-lhes uma melhor qualidade de vida. Sua intervenção é sempre baseada numa abordagem holística e centrada no cliente. A valorização da vida e da autonomia da pessoa com doença fora de possibilidade de cura é o foco principal do trabalho da Terapia Ocupacional em Cuidados Paliativos. Pacientes e terapeutas ocupacionais devem avaliar quais tarefas são necessárias, possíveis dentre de suas capacidades remanescentes e que dão sentido à vida e possibilitam o desempenho de seus papéis ${ }^{13}$.

A intervenção de Terapia Ocupacional nos Cuidados Paliativos busca criar possibilidades de ampliação da autonomia e das possibilidades do fazer, compreendendo as atividades como possibilidades de experiências de potência, permitindo o resgate de capacidades remanescentes, bem como a criação de projetos a serem realizados. Toda intervenção está voltada à permanência de atividades significativas no cotidiano do paciente e de sua família ${ }^{14}$. Ainda que sua história de vida pessoal sofra uma ruptura ${ }^{4}$, o cotidiano e as possibilidades de escolhas e/ou fazeres estejam limitados pelo adoecimento e tratamento, a vida não pode perder seu sentido.

Assim, este estudo tem por objetivo identificar e categorizar as intervenções desenvolvidas pelo terapeuta ocupacional com mulheres com câncer de mama em Cuidados Paliativos, atendidas pelo serviço de Cuidados Paliativos de um hospital público, universitário, de alta complexidade, localizado no interior do Estado de São Paulo.

\section{PROCEDIMENTOS METODOLÓGICOS}

Trata-se de um estudo retrospectivo, descritivo, baseado em análise documental, de abordagem qualitativa baseado na revisão dos dados sociodemográficos e clínicos de mulheres com câncer ginecológico, registrados no banco de dados, de um serviço hospitalar de Cuidados Paliativos, no período de agosto de 2010 a dezembro de 2012. Após a aprovação do projeto pelo Comitê de Ética em Pesquisa do hospital (Processo $\mathrm{n}^{\circ}$ 12559/2013), foram identificadas as pacientes com câncer ginecológico e foi realizada uma revisão em profundidade de dados de prontuários clínicos (ativos ou recuperados do arquivo morto) daquelas com câncer de mama atendidas pela Terapia Ocupacional. A partir dos relatos das intervenções realizadas pela terapia ocupacional, foram identificadas categorias emergentes que serão apresentadas nos resultados deste estudo.

A casuística inicial foi composta por 49 mulheres com câncer ginecológico, atendidas de agosto de 2010 a dezembro de 2012 pela equipe multiprofissional do serviço hospitalar de Cuidados Paliativos. Dentre elas, foram identificados os seguintes tipos de câncer:

1. Câncer de mama: 27 mulheres;

2. Câncer de útero: 15 mulheres;

3. Câncer de ovário: 4 mulheres;

4. Câncer de endométrio: 2 mulheres;

5. Câncer de vulva: 1 mulher.

A primeira etapa da coleta de dados se deu através de análise do banco de dados eletrônico do serviço de Cuidados Paliativos e das fichas de avaliação preenchidas pelos profissionais, onde constam informações referentes à data do Pedido de Interconsulta para o Grupo, data da avaliação, identificação do paciente e cuidados, diagnóstico e prognóstico, tratamentos já realizados, funcionalidade (avaliada pela Escala de Desempenho de Karnofsky - KPS), medicamentos em uso, sintomas (avaliados pela Edmonton Symptom Assessment System 
- ESAS), exames laboratoriais, data de óbito e tempo de seguimento do grupo.

Na sequência do trabalho, foram identificadas 27 mulheres com câncer de mama, sendo que, dentre elas, 23 mulheres compuseram a casuística final do estudo por terem sido atendidas pela Terapia Ocupacional. A análise dos atendimentos realizados permitiu o levantamento de categorias (definidas a posteriori) relativas às intervenções de Terapia Ocupacional com mulheres com câncer de mama.

\section{RESULTADOS}

Os dados sociodemográficos e ocupacionais dos sujeitos da pesquisa são apresentados na Tabela 1. O Grupo 1 é composto por mulheres com câncer de mama (27 ou 55,1\% da casuística total) e do Grupo 2 é composto por mulheres com outros tipos de câncer ginecológico, como de útero, de ovário, de endométrio e de vulva ( 22 ou $44,9 \%$ da casuística total). Das 49 mulheres estudadas, 40 já haviam morrido no momento da coleta de dados (novembro de 2013).

Tabela 1 - Caracterização Sociodemográfica e ocupacional das mulheres com câncer ginecológico (G1) e câncer de mama (G2)

\begin{tabular}{|c|c|c|c|c|c|c|}
\hline & \multicolumn{2}{|c|}{$\begin{array}{c}\text { Grupo } 1 \\
\text { (câncer de mama) }\end{array}$} & \multicolumn{2}{|c|}{$\begin{array}{c}\text { Grupo } 2 \\
\text { (outros tipos de câncer } \\
\text { ginecológico) }\end{array}$} & \multicolumn{2}{|c|}{ Total } \\
\hline & $(\mathbf{N})$ & $\%$ & $(\mathrm{~N})$ & $\%$ & $(\mathbf{N})$ & $\%$ \\
\hline \multicolumn{7}{|l|}{ Sexo } \\
\hline Feminino & 27 & 55,10 & 22 & 44,9 & 49 & 100 \\
\hline Masculino & 0 & 0 & 0 & 0 & 0 & 0 \\
\hline \multicolumn{7}{|l|}{ Estado civil } \\
\hline Solteira & 7 & 14,28 & 06 & 12,25 & 13 & 26,53 \\
\hline Casada & 10 & 20,41 & 06 & 12,25 & 16 & 32,66 \\
\hline Divorciada & 02 & 4,08 & 04 & 8,16 & 06 & 12,24 \\
\hline Viúva & 05 & 10,20 & 02 & 4,08 & 07 & 14,28 \\
\hline Separada & 02 & 4,08 & 03 & 6,12 & 05 & 10,20 \\
\hline Amasiada & 01 & 2,05 & 01 & 2,04 & 02 & 4,09 \\
\hline \multicolumn{7}{|l|}{ Idade } \\
\hline $30-40$ & 02 & 4,08 & 01 & 2,04 & 03 & 6,12 \\
\hline $41-50$ & 05 & 10,20 & 02 & 4,08 & 07 & 14,28 \\
\hline $51-60$ & 04 & 8,16 & 09 & 18,38 & 13 & 26,54 \\
\hline $61-70$ & 10 & 20,41 & 05 & 10,20 & 20 & 30,61 \\
\hline $71-80$ & 05 & 10,20 & 02 & 4,08 & 07 & 14,28 \\
\hline 81-90 & 01 & 2,05 & 03 & 6,12 & 04 & 8,17 \\
\hline \multicolumn{7}{|l|}{ Escolaridade } \\
\hline Analfabeto & 02 & 4,08 & 01 & 2,04 & 03 & 6,15 \\
\hline Fundamental incompleto & 09 & 18,38 & 08 & 16,34 & 17 & 34,66 \\
\hline Fundamental completo & 05 & 10,20 & 03 & 6,12 & 08 & 16,32 \\
\hline Médio incompleto & 02 & 4,08 & 01 & 2,04 & 03 & 6,15 \\
\hline Médio completo & 04 & 8,16 & 04 & 8,16 & 08 & 16,33 \\
\hline Superior incompleto & 02 & 4,08 & 01 & 2,04 & 03 & 6,15 \\
\hline Superior completo & 03 & 6,12 & 04 & 8,16 & 07 & 14,28 \\
\hline \multicolumn{7}{|l|}{ Ocupação } \\
\hline Do lar & 15 & 30,61 & 10 & 20,40 & 25 & 51,01 \\
\hline Autônoma & 06 & 12,24 & 04 & 8,16 & 10 & 20,40 \\
\hline Professora & 03 & 6,12 & 00 & 00 & 03 & 6,12 \\
\hline Profissional da saúde & 02 & 4,08 & 00 & 00 & 02 & 4,08 \\
\hline Outros (serviços gerais) & 01 & 2,05 & 08 & 16,34 & 09 & 18,39 \\
\hline \multicolumn{7}{|l|}{ Religião } \\
\hline Católico & 12 & 24,49 & 12 & 24,50 & 24 & 48,99 \\
\hline Protestante & 08 & 16,32 & 03 & 6,12 & 11 & 22,44 \\
\hline Espírita & 02 & 4,08 & 04 & 8,16 & 06 & 12,24 \\
\hline Outra & 01 & 2,05 & 00 & 00 & 01 & 2,05 \\
\hline Sem religião definida & 04 & 8,16 & 01 & 2,04 & 05 & 10,20 \\
\hline Sem resposta & 00 & 00 & 02 & 4,08 & 02 & 4,08 \\
\hline Sub-total & 27 & 55,10 & 22 & 44,9 & 49 & 100 \\
\hline
\end{tabular}


A faixa etária prevalente dentre as mulheres com câncer de mama (considerando a idade no momento da avaliação) foi de 61 a 70 anos (20,41\%), 34,7\% estavam internadas e acompanhadas durante a internação, $20,41 \%$ eram casadas e $18,38 \%$ não concluíram o ensino fundamental. Quanto à ocupação, a maior parte $(30,61 \%)$ das mulheres era do lar (atividades domésticas) e 12,24\% exerciam atividade autônoma. Dentre as mulheres com outros tipos de câncer, destaca-se o fato de que há o mesmo número de mulheres solteiras e casadas $(12,25 \%)$, a faixa etária prevalente foi de 51 a 60 anos (18,38\%), 20,40\% estavam sendo acompanhadas durante a internação, 16,34\% das mulheres não concluíram o ensino fundamental e em relação à ocupação, 20,40\% eram do lar.

Em relação aos resultados obtidos através da escala de Karnofsky (Tabela 2), 22,46\% das mulheres com câncer de mama estavam com KPS entre $10 \%$ e $40 \%$ e $20,40 \%$ estavam com a pontuação de KPS entre $50 \%$ e $70 \%$, enquanto que dentre as mulheres com outros tipos de câncer ginecológico 16,32\% estavam com KPS entre 10 \%e $40 \%, 16,33 \%$ estavam com KPS entre $50 \%$ e $70 \%$ e $12,25 \%$ com KPS de $80 \%$.

Tabela 2 - Caracterização clínica das mulheres com câncer ginecológico e câncer de mama

\begin{tabular}{|c|c|c|c|c|c|c|}
\hline \multirow[b]{2}{*}{ KPS (\%) } & \multicolumn{2}{|c|}{$\begin{array}{c}\text { Grupo } 1 \\
\text { (câncer de mama) }\end{array}$} & \multicolumn{2}{|c|}{$\begin{array}{l}\text { Grupo } 2 \\
\text { (outros tipos de câncer } \\
\text { ginecológico) }\end{array}$} & \multicolumn{2}{|c|}{ Total } \\
\hline & (N) & $\%$ & $(\mathrm{~N})$ & $\%$ & $(\mathrm{~N})$ & $\%$ \\
\hline 100 & 00 & 00 & 00 & 00 & 00 & 00 \\
\hline 90 & 02 & 4,08 & 00 & 00 & 02 & 4,08 \\
\hline & 00 & 00 & 06 & 12,25 & 06 & 12,25 \\
\hline 70 & 05 & 10,20 & 00 & 00 & 05 & 10,20 \\
\hline 00 & 03 & 6,12 & 06 & 12,25 & 09 & 18,37 \\
\hline 0 & 02 & 4,08 & 02 & 4,08 & 04 & 8,16 \\
\hline 40 & 10 & 20,41 & 05 & 10,20 & 15 & 30,61 \\
\hline 50 & 00 & 00 & 00 & 00 & 00 & 00 \\
\hline 20 & 01 & 2,05 & 00 & 00 & 01 & 2,05 \\
\hline 10 & 00 & 00 & 00 & 00 & 00 & 00 \\
\hline Seml Iesposta & 04 & 8,16 & 03 & 6,12 & 07 & 14,28 \\
\hline Sub-total & 27 & 55,10 & 22 & 44,9 & 49 & 100 \\
\hline
\end{tabular}

De acordo com a escala de sintomas Edmonton Symptom Assessment System - ESAS (Tabela 3), as mulheres com câncer de mama apresentaram, predominantemente, os seguintes sintomas: dor $(51,86 \%)$, náusea $(81,49 \%)$, mal estar e falta de ar $(66,67, \%)$, falta de apetite $(42,30 \%)$ e ansiedade $(59,25 \%)$ avaliados como leves; sonolência $(44,45 \%)$ avaliada como moderada; cansaço (40,74\%) avaliado como forte. Em relação às mulheres com outros tipos de câncer ginecológico (Tabela 4) os sintomas que se destacaram foram: náusea $(68,18 \%)$, depressão $(50 \%)$, ansiedade $(50 \%)$, sonolência $(72,72 \%)$, falta de apetite $(50 \%)$, mal estar $(54,54 \%)$ e falta de ar $(72,72)$ classificados como leves; cansaço (50\%) avaliado como moderado. 
Faria NC, De Carlo MRP.A atuação da terapia ocupacional com mulheres com câncer. Rev Ter Ocup Univ São Paulo. 2015 set.-dez.;26(3):418-27.

Tabela 3 - Resultados da Escala Edmonton Symptom Assessment System de mulheres com câncer de mama (N=27)

\begin{tabular}{|c|c|c|c|c|c|c|c|c|}
\hline & $\begin{array}{l}\text { Leve } \\
(0-3)\end{array}$ & $\%$ & $\begin{array}{c}\text { Moderada } \\
(4-7)\end{array}$ & $\%$ & $\begin{array}{l}\text { Forte } \\
(8-10)\end{array}$ & $\%$ & Total & $\%$ \\
\hline Dor & 14 & 51,86 & 10 & 37,03 & 3 & 11,11 & 27 & 100 \\
\hline Cansaço & 6 & 22,22 & 10 & 37,03 & 11 & 40,75 & 27 & 100 \\
\hline Náusea & 22 & 81,49 & 4 & 14,81 & 1 & 3,70 & 27 & 100 \\
\hline Depressão & 12 & 44,45 & 9 & 33,33 & 6 & 22,22 & 27 & 100 \\
\hline Ansiedade & 16 & 59,25 & 8 & 29,64 & 3 & 11,11 & 27 & 100 \\
\hline Sonolência & 10 & 37,03 & 12 & 44,45 & 5 & 18,52 & 27 & 100 \\
\hline Falta de apetite & 11 & 42,30 & 6 & 23,09 & 9 & 34,61 & $26(*)$ & 100 \\
\hline Mal estar & 18 & 66,67 & 4 & 14,81 & 5 & 18,52 & 27 & 100 \\
\hline Falta de ar & 18 & 66,67 & 7 & 25,93 & 2 & 7,40 & 27 & 100 \\
\hline
\end{tabular}

(*) Faltou o dado de um paciente - não preenchido pelos profissionais

Gráfico 1 - Categorias relativas às intervenções de Terapia Ocupacional com mulheres com câncer de mama

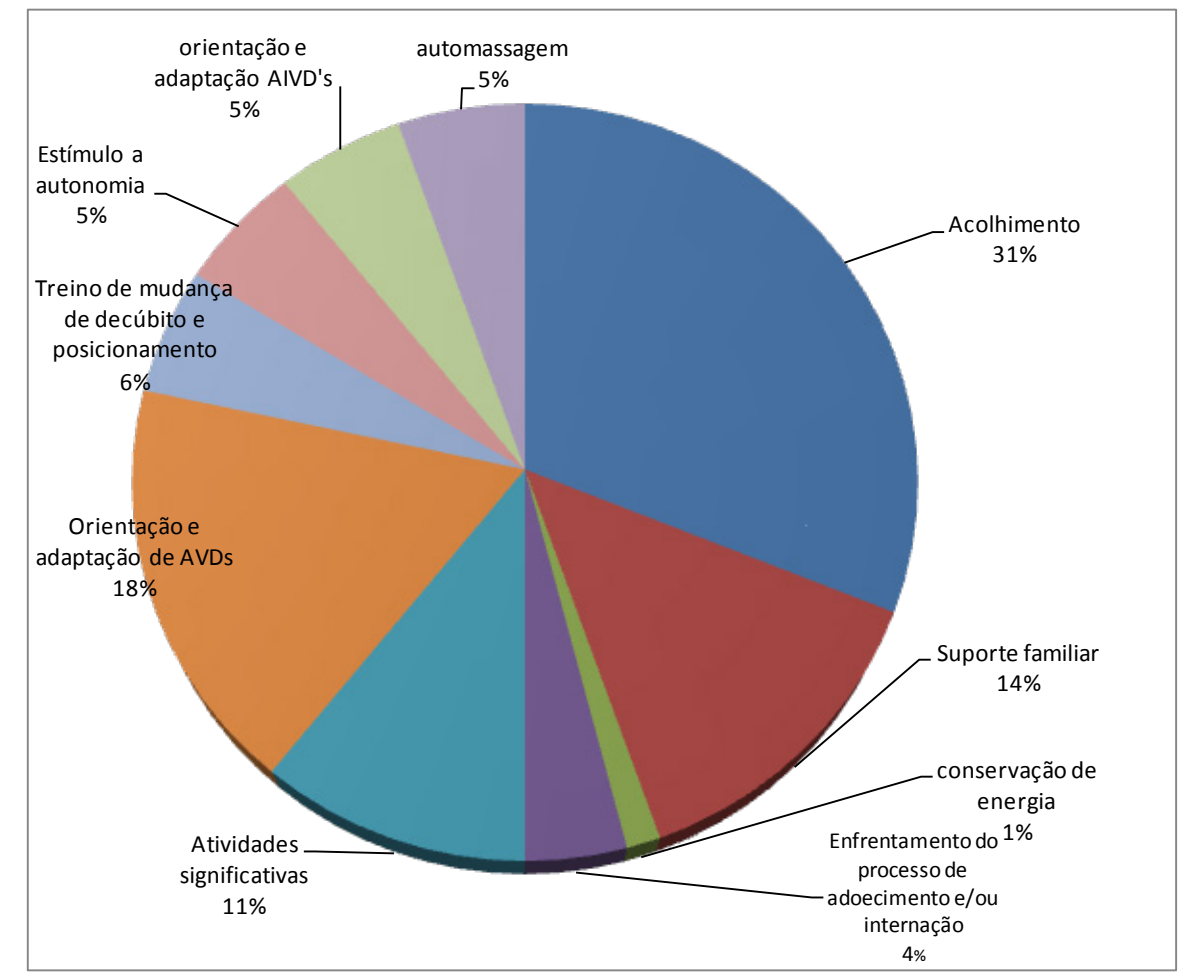


Os atendimentos de Terapia Ocupacional às mulheres com câncer de 'mama variaram de 1 a 4 sessões. As intervenções foram classificadas em 10 categorias (apresentadas no Gráfico 1), de acordo com os procedimentos realizados com cada paciente sendo que em cada atendimento pode ter sido realizado mais de um procedimento, o quais serão discutidos a seguir.

\section{DISCUSSÃO}

Conforme os dados sociodemográficos e clínicos, coletados através das avaliações realizadas pela equipe de Cuidados Paliativos, observou-se um número expressivo de mulheres com câncer de mama com baixa funcionalidade $(K P S)$, o que indica dificuldades para cuidar de si próprias dentre outros aspectos. Observou-se também considerável carga de sintomas (ESAS), ainda que predominantemente leves e moderados, com exceção do cansaço, avaliado como forte por mulheres com câncer de mama e moderado por aquelas com outros tipos de câncer ginecológico.

Por meio da análise dos registros dos prontuários das 27 mulheres com câncer de mama encaminhadas ao grupo de Cuidados Paliativos, no período do estudo, 23 pacientes foram atendidas pela terapeuta ocupacional da equipe. Quatro pacientes não foram atendidas pela terapeuta ocupacional por motivo de óbito antes da primeira.

As intervenções foram assim categorizadas:

1. Acolhimento: Segundo o Ministério da Saúde ${ }^{15}$, acolhimento é uma diretriz da Política Nacional de Humanização (PNH), que deve fazer parte de todos os encontros dos serviços de saúde e deve ser realizado por todos os membros da equipe. É uma postura ética que implica na escuta do usuário em suas queixas, no reconhecimento do seu protagonismo no processo de saúde e adoecimento e na responsabilização pela resolução, com ativação de redes de compartilhamento de saberes. Acolher é um compromisso de resposta às necessidades dos cidadãos que procuram os serviços de saúde. Neste estudo, o acolhimento foi realizado com todas as pacientes atendidas pela terapeuta ocupacional ${ }^{15}$;

2. Orientação e adaptação para realização de Atividades de vida diária (AVDs): Atividades orientadas para o cuidado do indivíduo para com seu próprio corpo, que são fundamentais para viver no mundo social e que permitem a sobrevivência e o bem-estar. Neste estudo, as ações voltadas para orientação de cuidados gerais e adaptação de atividades de vida diária (AVDs) foram realizadas com 13 pacientes com objetivo de possibilitar maior independência funcional e ocupacional ao paciente, promovendo consequente melhora da autoestima, bemestar e qualidade de vida;

3. Orientação e adaptação de atividades instrumentais de vida diária (AIVD's): Atividades que apóiam a vida diária dentro de casa e na comunidade que, frequentemente, requerem maior complexidade nas interações do que o autocuidado nas AVDs. Neste estudo, a terapeuta ocupacional utilizou tais orientações e adaptações com 4 pacientes, a fim de promover melhor desempenho ocupacional e orientar quanto a adaptação do ambiente doméstico, com eliminação de barreiras arquitetônicas e modificações estruturais e ambientais;

4. Orientação e suporte familiar: 10 familiares e/ ou cuidadores foram sujeitos de intervenções do terapeuta ocupacional, tendo sido orientados em relação à facilitação da comunicação com o paciente, auxiliados na reorganização da dinâmica familiar devido ao adoecimento, e na expressão de sentimentos e expectativas relacionados ao prognóstico e no processo de elaboração do luto;

5. Realização de atividades significativas: estratégia empregada com 8 pacientes, a partir do relato de suas histórias de vida, em que foram percebidas rupturas geradas pelo processo de adoecimento. $\mathrm{O}$ resgate de tais atividades auxilia na adesão ao tratamento, na facilitação do vínculo terapêutico, na motivação para a reestruturação do cotidiano e da vida ocupacional, seja durante a internação, nas consultas ambulatoriais ou nos atendimentos domiciliares;

6. Treino de mudanças de decúbito e posicionamento: esses procedimentos foram realizados com 4 pacientes e familiares a fim de proporcionar maior funcionalidade, conforto, auxílio no controle de dor e prevenção de úlceras por pressão e/ou deformidades;

7. Estímulo à autonomia: foi relatado nos atendimentos realizados com 4 pacientes, tendo sido fundamental no processo de adaptação e aceitação dos novos papéis ocupacionais e manutenção da vida ativa, ainda que dentro das limitações impostas pelo adoecimento;

8. Orientação sobre automassagem: procedimentos realizado com 4 pacientes, com o objetivo de favorecer a circulação sanguínea do braço do lado operado e, com isso, prevenir edemas; 
9. Auxílio no enfrentamento do processo de adoecimento e hospitalização: esse tipo de intervenção ocorreu com 3 pacientes, que apresentaram dificuldades para desenvolver estratégias adaptativas e de enfrentamento do processo de adoecimento e internação. Envolveram orientações e proposições reflexivas relacionadas a novas possibilidades de organização da vida ocupacional, objetivando minimizar os sofrimentos vivenciados;

10. Orientação sobre conservação de energia: tal procedimento foi realizado com 1 paciente, com o objetivo de que ele pudesse realizar suas atividades com menor gasto energético, visando equilibro entre repouso e atividade e permitindo melhorar o desempenho na realização de AVD's, AIVD's, atividades de trabalho, lazer e participação social.

A atuação da terapeuta ocupacional baseou-se nos princípios da Prática Centrada no Cliente, o que condiz com a revisão da literatura realizada acerca da atuação da Terapia Ocupacional com pacientes oncológicos em Cuidados Paliativos. Neste sentido, a assistência em Terapia Ocupacional contribui para a saúde dos indivíduos hospitalizados, não levando em conta somente a doença e a hospitalização, mas considerando também questões psicossociais e espirituais. Por considerar a individualidade e singularidade do paciente, o terapeuta ocupacional pode estimular a realização de atividades especificas e/ou exercícios selecionados dentro do próprio espaço hospitalar e, dessa maneira trabalhar todos os aspectos que influenciam de forma significativa a vida do paciente, de modo a atender as necessidades emergentes ${ }^{16,17}$.

O foco central do trabalho da terapeuta ocupacional em Cuidados Paliativos é a prevenção do sofrimento e a promoção do bem-estar e qualidade de vida na vida cotidiana, proporcionando ao paciente o direito de ter uma vida significativa e produtiva, ainda que com limitações decorrentes da evolução da doença. As atividades significativas proporcionam o resgate da autonomia e da autoestima, do desejo de manter-se ativo e participativo, além de auxiliar para um melhor enfrentamento do adoecimento e da internação ${ }^{12}$.

É importante ressaltar que, na fase final de vida, deve haver mudança de foco do tratamento, sendo que a reorganização da rotina e a diminuição dos estímulos podem ser maneiras de propiciar maior conforto ao paciente. Em alguns casos, é possível manter as atividades significativas de acordo com o desejo do paciente. Na preparação para a morte, os terapeutas ocupacionais podem auxiliar no processo de fechamento, ajudando os pacientes a voltarem para casa e se despedirem dos seus familiares, valorizando a vida remanescente do indivíduo e ajudando-o a viver o presente, reconhecendo seu direito de autodeterminação e assistindo-o na aproximação da morte ${ }^{18}$

No acompanhamento familiar, pode-se ajudar nas despedidas, na expressão de sentimentos e emoções e na abertura de novos canais de comunicação. $O$ acompanhamento pós-óbito é parte integrante da assistência terapêutico ocupacional em Cuidados Paliativos, especialmente através de ligações telefônicas ou visitas de luto, em conjunto com outros profissionais ${ }^{14}$.

As intervenções relatadas neste estudo favorecem a funcionalidade do paciente, promovendo autonomia na realização das atividades de vida diária, instrumentais, de lazer e sociais, aumentando a possibilidade de manter-se ativo até o final da vida. Os recursos adaptativos foram utilizados pela terapeuta ocupacional como forma de maximizar a independência do paciente em suas AVD's, AIVD's e atividades significativas, além de favorecer no processo de enfrentamento ${ }^{19}$

Os atendimentos aos familiares ocorreram mediante a observação do sofrimento e desestruturação familiar diante do adoecimento e da possibilidade da morte de um de seus membros. O cuidado, a escuta e o acolhimento das demandas próprias dos familiares e cuidadores é parte integrante e fundamental na assistência terapêutico ocupacional. Desta forma, foi possível visualizar que as intervenções desenvolvidas com os familiares encontram respaldo na literatura, trabalhando aspectos psicossociais, possibilitando a expressão dos sentimentos e expectativas diante do prognóstico e processo de elaboração do luto ${ }^{14}$.

Além disso, percebeu-se a necessidade de orientar os familiares e cuidadores acerca dos estímulos ao paciente e de treiná-los para que sejam facilitadores da independência nas atividades de vida diária, assim como para a realização dos cuidados exigidos, como o manejo do membro operado, mudança de decúbito e de posicionamento, e na execução de suas atividades diárias ${ }^{19}$.

O paciente oncológico deve ser assistido por uma equipe multiprofissional desde o diagnóstico de uma doença potencialmente fatal, nos diversos contextos de atendimentos: pré internação, internação, ambulatório, hospital-dia, casa de apoio e unidade de retaguarda ou hospice, assim como em visita domiciliária. A Terapia Ocupacional atua em todos esses locais e em diferentes situações e períodos de tratamento, abrangendo tanto o paciente, como seus familiares/cuidadores, inclusive na preparação para a alta ou no processo de óbito. Se a pessoa adoecida e seus familiares conseguirem desenvolver um estilo adaptativo de vida, melhor será sua qualidade de 
vida, maior será a aceitação das próprias limitações, melhor será o processo de enfrentamento e o desenvolvimento de pensamentos positivos e mais afirmativos sobre o futuro e inclusive sobre o final da vida ${ }^{20}$.

\section{CONSIDERAÇÕES FINAIS}

Segundo os princípios dos Cuidados Paliativos, a pessoa que tem uma doença potencialmente fatal e está "fora de possibilidades de cura" necessita de um cuidado personalizado e um sistema de apoio durante toda a evolução de uma doença, de modo que o possa viva tão ativamente quanto possível, através do engajamento em atividades significativas, até a sua morte ${ }^{14}$.

Os profissionais de terapia ocupacional entendem o envolvimento ativo em ocupações a partir de uma perspectiva holística e recorrem a todos os aspectos das dimensões humanas e de desempenho ocupacional durante suas intervenções. Alcançar a saúde, bem-estar e participação social por meio das ocupações é a afirmação abrangente que descreve os domínios e processos de terapia ocupacional em seu sentido mais amplo ${ }^{21}$.

Porém, com o adoecimento e a internação, devido a doenças como o câncer, ocorre uma série de mudanças no cotidiano e o tratamento passa a ocupar uma grande parte da rotina e das preocupações tanto do paciente como de seus familiares. O terapeuta ocupacional pode desempenhar um papel significativo no cuidado multiprofissional do paciente com câncer, a fim de que ele possa atingir seus objetivos e melhorar sua qualidade de vida ${ }^{22}$. O trabalho em equipe torna possível oferecer uma assistência de melhor qualidade, de maneira que pacientes e familiares sejam adequadamente acolhidos e cuidados ${ }^{14,23}$.

O terapeuta ocupacional pode proporcionar escuta ativa e acolhimento ao paciente e sua família, a fim de identificar as principais demandas que estão interferindo de maneira significativa na sua vida, no desempenho de papéis e atividades cotidianas. Durante o processo terapêutico ocupacional é preciso compreender as mudanças nas condições clinicas, procedimentos já realizados e prognóstico, além dos significados atribuídos por pacientes e familiares a todo esse contexto de vida, marcado por mudanças físicas, emocionais, psicológicas, sociais e espirituais ${ }^{24}$.

Este trabalho possibilitou a apresentação de práticas desenvolvidas pela terapia ocupacional em cuidados paliativos, percebendo-se contribuições relacionadas à manutenção da autonomia, prevenção de incapacidades, melhora no desempenho ocupacional e da capacidade funcional, influenciando na qualidade de vida e possibilitando que o sujeito viva e possa morrer com mais conforto e dignidade, no hospital ou na residência. Assim, pode minimizar sofrimentos e desconfortos por meio de intervenções no controle de dor e sintomas, na manutenção da "vida ativa" com o máximo de autonomia e independência ${ }^{25}$.

Considerando a atuação em Cuidados Paliativos como uma prática ainda recente do terapeuta ocupacional, é importante o investimento na elaboração de novas pesquisas e publicações, para que se possa promover discussões voltadas ao aprimoramento técnico-científico da profissão neste campo de conhecimentos e de atuação.

CONFLITO DE INTERESSES: Não há.

AGRADECIMENTOS: À Fundação para o Desenvolvimento Administrativo (FUNDAP); Ao Hospital das Clínicas da Faculdade de Medicina de Ribeirão Preto - USP; Ao Grupo de Cuidados Paliativos do Hospital das Clínicas da Faculdade de Medicina de Ribeirão Preto (HCFMRP-USP).

\section{REFERENCIAS}

1. Instituto Nacional do Câncer. Estimativa 2014. Disponivel em: http://www.inca.gov.br/estimativa/2014/index.asp?ID=2.

2. Instituto Nacional do Câncer. Estimativas. Disponível em: http://www2.inca.gov.br/wps/wcm/connect/tiposdecancer/ site/home/mama.

3. Elsner VR, Trentin RP, Horn CC. Efeito da hidroterapia na qualidade de vida de mulheres mastectomizadas. Rev Arq Ciên Saúde. 2009;16 (2):67-71. Disponível em: http://repositorio-
racs.famerp.br/racs_ol/vol-16-2/ID330.pdf.

4. Pengo MMSB, Santos WA. O papel do terapeuta ocupacional em oncologia In: De Carlo MMRP, Luzo MCM. Terapia ocupacional: reabilitação física e contextos hospitalares. São Paulo: Roca; 2004. p.233-55.

5. Almeida RA. Impacto da mastectomia na vida da mulher. Rev Soc Bras Psicol Hosp. 2006;9(2):99-113. Disponível em: http://pepsic.bvsalud.org/pdf/rsbph/v9n2/v9n2a07.pdf. 
6. Pereira SG, Rosenhein DP, Bulhosa MS, Lunardi VL, Filho WDL. Vivências de cuidados da mulher mastectomizada: uma pesquisa bibliográfica. Rev Bras Enferm. 2006;59(6):791-805. http://dx.doi.org/10.1590/S0034-71672006000600013.

7. Almeida AM, Mamede MV, Panobianco MS, Prado MAS, Clapis, M.J. Construindo o significado da recorrência da doença: a experiência de mulheres com câncer de mama. Rev Latino-amer Enferm. 2001;9(5):63-90. Disponível em: http:// www.scielo.br/pdf/rlae/v9n5/7800.pdf.

8. Bittencourt JFV, Cadete MMM. Vivências da mulher a ser mastectomizada: esclarecimentos e orientações. Rev Bras Enferm. 2002;55(4):420-30. http://www.scielo.br/pdf/reben/ v55n4/v55n4a10.pdf.

9. Rossi L, Dos Santos MA. Repercussões psicológicas do adoecimento e tratamento em mulheres acometidas pelo câncer de mama. Psicol Ciên Profissão. 2003;23(4):32-41. Disponível em: http://www.scielo.br/pdf/pcp/v23n4/v23n4a06.pdf.

10. Pessini L. Cuidados Paliativos: perspectivas contemporâneas. Rev Bras Cuidados Paliativos. 2008;1(1):25-9. Disponível em: http://www.cuidadospaliativos.com.br/img/din/file/RBCP1. pdf.

11. World Health Organization. National cancer control programmes: policies and managerial guidelines. 2a ed. Geneva: WHO; 2002. Available from: http://www.who.int/ cancer/media/en/408.pdf.

12. De Carlo MMRP, Queiroz MEG, organizadores. Dor e cuidados paliativos - terapia ocupacional e interdisciplinaridade. São Paulo: Roca; 2008.

13. Cooper J. Occupational therapy in oncology and palliative care. 2nd ed. Chichester: Wiley; 2006.

14. Othero MB. O papel do terapeuta ocupacional na equipe. In: Carvalho RT, Parsons HA. Manual de cuidados paliativos ANCP. 2a ed. ampl. atual. Porto Alegre: Sulina; 2012. p.361-3.

15. Brasil. Ministério da Saúde. Política Nacional de Humanização. Acolhimento. Disponível em: http://bvsms.saude.gov.br/bvs/ dicas/167acolhimento.html.
16. Sumsion T. Prática baseada no cliente na Terapia Ocupacional: guia para implementação. São Paulo: Roca; 2003.

17. Silva ACC, Giuardinetto ARSB. Políticas públicas em oncologia: refletindo sobre a atuação da terapia ocupacional. Rev Ter Ocup Univ São Paulo. 2012;23:297-308. Disponível em: http://www.revistas.usp.br/rto/article/viewFile/55646/59106.

18. Bye R. When clients are dying, occupational therapy perspective. Occup Ther Res.1998;18:3-24.

19. Silva NP, Kovacs, ACTB. Terapia ocupacional na atenção domiciliar a pacientes com dor e em cuidados paliativos. In: De Carlo MMRP, Queiroz MEG. Dor e cuidados paliativos: terapia ocupacional e interdisciplinaridade. São Paulo: Roca; 2008. p.307-325.

20. Bigatão MR, Mastropietro AP, De Carlo MMRP. Terapia ocupacional em oncologia - a experiência do hospital das clínicas da Faculdade de Medicina de Ribeirão Preto da Universidade de São Paulo. In: Othero B. Terapia ocupacional práticas em oncologia. São Paulo: Roca; 2010. p.302-22.

21. AOTA. Estrutura da prática da Terapia Ocupacional: domínio \& processo 3a ed. Rev Ter Ocup Univ São Paulo. 2015;26(ed. esp.):1-49. http://dx.doi.org/10.11606/issn.2238-6149. v26iespp1-49

22. Vockins H. Occupational therapy intervention with patients with breast cancer: a survey. Eur J Cancer Care (Engl.). 2004;13:45-52. doi: 10.1111/j.1365-2354.2004.00443.x

23. Ferrari MAC. Lazer e ocupação do tempo livre na terceira idade. In: Papaleo Netto M. Gerontologia: a velhice e o envelhecimento em visão globalizada. São Paulo: Atheneu; 2005. p. $98-105$.

24. Ferrer AL, Santos WA. Terapia ocupacional na atenção a pacientes com dor oncológica e cuidados paliativos. In: De Carlo MMRP, Queiroz MEG. Dor e cuidados paliativos: terapia ocupacional e interdisciplinaridade. São Paulo: Roca; 2008. p.148-66.

25. Queiroz MEG. Atenção em cuidados paliativos. Cad Ter Ocup UFSCar (São Carlos). 2012;20:203-5. http://dx.doi. org/10.4322/cto.2012.021. 